\title{
Investigating the Transfer of Drug Particulate onto Evidence Packaging During Routine Case Analysis
}

\author{
Edward Sisco ${ }^{*}$, Elizabeth L. Robinsona, Rebecca Mead ${ }^{b}$, Charles R. Miller IVc \\ aNational Institute of Standards and Technology \\ ${ }^{b}$ Vermont Forensic Laboratory \\ "Maryland State Police Forensic Sciences Division \\ edward.sisco@nist.gov, 301-975-2093
}

\section{Highlights}

- Drug residues on evidence packaging were measured prior to and after case analysis.

- In nearly all instances, an increase in the amount of residue on the packaging was observed.

- Similar results were obtained for items repackaged in the original Kapak and in a new Kapak.

- Residue on seized drug packaging continued to be an excellent predictor of the contents.

\section{Abstract}

The presence of drug residue and drug background in a forensic context continues to be of interest for a variety of reasons ranging from its potential use as a means for presumptive identification to ensuring the safety and wellbeing of drug chemists. While prior work has studied the presence of these residues on laboratory surfaces and on drug evidence, the contribution of residue on the exterior of drug packaging from the analysis process itself has not yet been studied. This work aims to qualitatively and quantitively identify what, if any, effect the analysis of drug evidence has on the drug residue levels on the exterior of the evidence packaging. Using wipe collection techniques, samples from the exterior of drug evidence packaging were taken prior to opening cases and after repackaging to measure changes in residue composition and mass. A total of 64 submissions were analyzed, and an increase in drug residue mass was observed $85.5 \%$ of the time. After analysis and repackaging, $95 \%$ of packages had detectable drug residue on their exterior even though some of the cases were repackaged into new bags. Drug residue masses on the exterior of drug packaging were found to be as high as tens of micrograms. The presence of drug residue on the exterior of drug evidence packaging is expected given the collection and analysis procedures, therefore potential ways to minimize these levels are currently being studied. The presence of these residues is an important factor to consider when developing protocols for the entire evidence handling process and its impact on personnel - from evidence handling technicians to crime scene technicians to submitting officers.

Keywords: Drug Evidence; Residue; Background; Exposure; Opioids; Safety; DART-MS

\section{Introduction}

Given the potency of many emerging drugs and novel psychoactive substances, the health and safety of forensic practitioners, evidence handling technicians, crime scene technicians, and others in the forensic analysis chain continues to be of major concern. While there has been little reported in terms of exposure of forensic chemists to controlled substances, recent reports have identified that such exposure can occur[1]. Similar reports relating to clandestine laboratory chemists and emergency responders also highlight the potential exposure risks that emerging drugs, such as synthetic opioids, present[2,3]. One potential route, or indicator, of exposure is residual drug particulate that can be found on surfaces and items throughout the laboratory. A number of studies have shown that drug residues are ubiquitous in drug chemistry units of forensic laboratories[4-6] and in evidence handling areas of police stations[7,8]. Other studies, which have visualized the evidence handling process, have demonstrated that aerosolization of drug particulate while handling powders is one of the main drivers for the presence of residue on surfaces[9,10]. Aerosolization of particulates is a problem that is not unique to forensic drug 
chemistry and has been demonstrated in other fields such as pharmaceutical production[11], medicine[12-14], and food science[15].

Understanding the presence and prevalence of drug background in a forensic environment, as well as the processes that may contribute to or prevent accumulation of residue, is critical to better inform the development of best practices. Several best practices have been established to increase safety of drug chemists and evidence handling techniques which outline ways to prevent exposure through inhalation or accidental contact. Suggested best practices always recommend that personnel wear gloves when handling drug evidence, regardless of whether the evidence is open or sealed. Wearing gloves when handling open drug evidence is a logical practice, but the use of gloves when handling sealed evidence may be less intuitive. Previous work has demonstrated that, frequently, drug residue is present even on the outer evidence packaging and may have been transferred onto the packaging through contaminated environments in which the evidence was packaged[16].

The study described here looks to take previous work one step further and examine whether or not opening and analyzing the drug evidence of a case in a forensic laboratory contributes to the drug residue on the outer evidence packaging. To do so, wipe samples of drug evidence packaging were taken prior to opening submissions and after re-packaging. Qualitative and quantitative measurements of the wipes were then made to establish the identities and quantities of drug residues on the exterior of the evidence packaging to better understand whether or not the act of opening, analyzing, and repackaging evidence leads to increases in the number and amounts of drugs present. The goal of this study was to provide tangible data to support the recommendation of glove use by those who are handling sealed drug evidence while also providing baseline measurements to better inform future studies focused on possible processes to reduce the transfer of residue during the analysis process.

\section{Materials and Methods}

Sampling Collection and Preparation

Collection of wipe samples from actual drug evidence submission was completed at the Vermont Forensic Laboratory and the Maryland State Police Forensic Sciences Division. Since the goal of this effort was to identify what, if any, drug residue is transferred onto the evidence packaging during the forensic analysis process, a sequential wiping process, outlined in Figure 1, was used. Dry meta-aramid wipes (DSA Detection, North Andover, MA, USA) were used for sample collection and stored, individually, in manilla envelopes after collection. Dry wipes were used to avoid smearing or dissolving important markings or signatures on the evidence packaging. Collection was completed using a unidirectional wiping pattern with a firm force.

\section{Samples}

A total of 64 individual evidence submissions were analyzed. The outer packaging for all submissions was a Kapak bag, and all submissions were repackaged into a Kapak bag. Two different repackaging protocols were used, depending on the laboratory, with submissions being repackaged into a new Kapak bag $(n=46)$ or being repackaged into the original Kapak bag $(n=18)$. Most submissions $(n=48)$ consisted of a single item, though 14 submissions had two items within the submission and two submissions had three items resulting in a total of 82 individual items and 82 inner packages. Of those items, the majority $(n=52)$ of the inner packaging were plastic 
bags, with an additional 22 glassine bags, three foil bags or folds, two suboxone packets, one paper bag, one screw cap vial, and one piece of currency. All but three of the items contained powder. Two items contained suboxone strips and one item contained residue on currency.

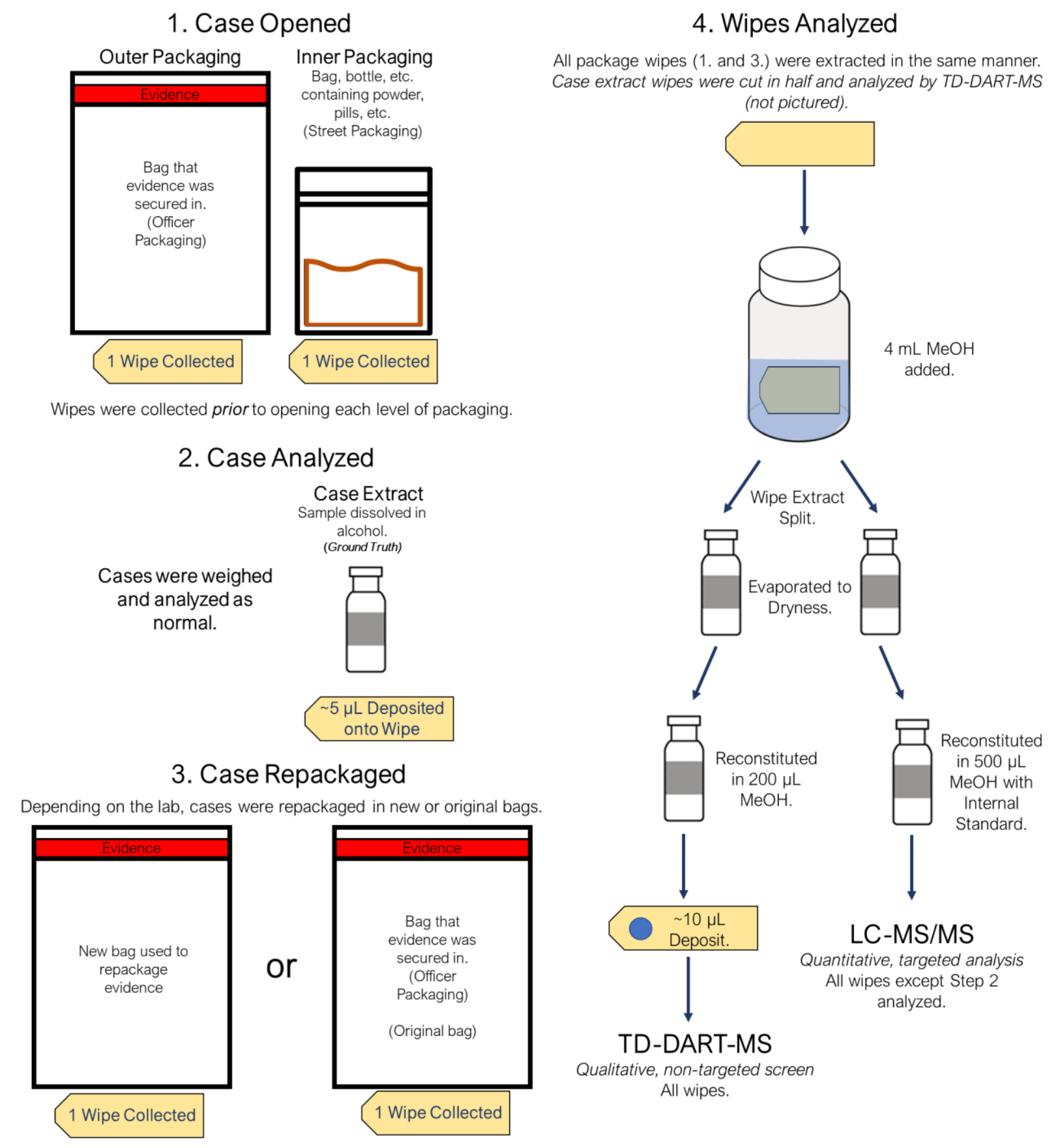

Wipes were collected after repackaging was completed.

Figure 1. Schematic of the sample collection and analysis procedure used for this study. Abbreviations for thermal desorption direct analysis in real time mass spectrometry (TD-DART-MS) and liquid chromatography tandem mass spectrometry (LC-MS/MS) are used.

For each case the following protocol was used. When submitted evidence was to be analyzed, the chemist first wiped the outer packaging, defined as the Kapak bag that the evidence was submitted in, and stored the wipe in a 
marked manilla envelope. The chemist then opened the outer packaging as they normally would and removed the contents, which consisted of one or more inner packages (glassine envelopes, pill bottles, etc.). To gain a further understanding of the ability of inner packaging residue to predict the drug contents, as shown in previous work[16], each inner package was also wiped by the chemist prior to opening. Separate wipes were used for each inner package and stored individually in marked manilla envelopes. Following this step (Figure 1, Step 1), chemists analyzed their cases as they normally would. When samples were prepared for analysis by gas chromatography mass spectrometry (GC-MS) an approximately $5 \mu \mathrm{L}$ aliquot of the evidence extract was placed on another wipe to provide a ground truth sample for analysis (Figure 1, Step 2). Once the chemist completed their analyses, the items were repackaged following each laboratory's protocols. For one laboratory, this involved re-packaging evidence in the original Kapak bag that the evidence was submitted in. The other laboratory repackaged items into a new Kapak bag. Once repackaging was completed, a final wipe of the outer packaging was taken, with the chemist using new gloves, and stored individually in a marked envelope (Figure 1, Step 3). Once the wipes were collected, they were extracted and analyzed using a combination of thermal desorption direct analysis in real time mass spectrometry (TD-DART-MS) and liquid chromatography tandem mass spectrometry (LC-MS/MS) for qualitative and quantitative purposes, respectively.

\section{Extraction of Wipes}

All outer packaging wipes were extracted in the same manner (Figure 1, Step 4), which first involved placing wipes in $10 \mathrm{~mL}$ glass vials and adding $4 \mathrm{~mL}$ methanol (Chromasolv-grade, Millipor-Sigma, St. Louis, MO, USA). After vortexing the vials at $50 \mathrm{~Hz}(3000 \mathrm{rpm})$ for $30 \mathrm{~s}$, the $4 \mathrm{~mL}$ was split into two $2 \mathrm{~mL}$ glass vials, one for qualitative and one for quantitative analysis. Both vials were evaporated to dryness by leaving the vials open in a fume hood for several days. Once evaporated, $200 \mu \mathrm{L}$ of methanol was added to the qualitative (TD-DART-MS) vial which was capped and vortexed. A $10 \mu \mathrm{L}$ aliquot was then pipetted onto a new meta-aramid wipe for analysis by TD-DARTMS. For samples with visible residue after evaporation, a deposit of $1 \mu \mathrm{L}$ to $2 \mu \mathrm{L}$ was used to prevent overloading the thermal desorption chamber. For the quantitative extract, once evaporated it was reconstituted in $500 \mu \mathrm{L}$ of methanol containing deuterated internal standards (cocaine-d3, fentanyl-d5, heroin- $d 9$, and methamphetamine-d5). Vials were capped, vortexed, and then loaded onto the autosampler for LC-MS/MS analysis. The wipe containing the evidence extract from Step 2 (Figure 1) was not extracted prior to qualitative analysis.

\section{Qualitative TD-DART-MS Analysis}

TD-DART-MS, which is described in detail elsewhere[17], was used as a non-targeted, qualitative screening tool and was chosen as it has excellent sensitivity, is rapid, and allows for direct analysis of wipes. The TD-DART-MS system used incorporated a JEOL JMS-T100LP mass spectrometer (JEOL USA, Peabody, MA, USA) coupled with a DART-SVP ion source (IonSense, Saugus, MA, USA), and a custom thermal desorption unit. All analyses were completed in positive ionization mode with a thermal desorber temperature of $265^{\circ} \mathrm{C}$, a nitrogen DART gas stream temperature of $400^{\circ} \mathrm{C}$, a DART grid electrode voltage of $+100 \mathrm{~V}$, and a Vapur flow rate of approximately $4 \mathrm{~L} \mathrm{~min}^{-1}$. Mass spectrometer settings included an orifice 1 voltage of $+20 \mathrm{~V}$, a ring lens and orifice 2 voltage of $+5 \mathrm{~V}$, an orifice temperature of $100^{\circ} \mathrm{C}$, and a peaks voltage (RF ion guide) of $+800 \mathrm{~V}$. A mass spectral scan range of $m / z 80$ 
to $m / z 600$ was used with a 2 scan s $^{-1}$ scan rate. Polyethylene glycol 600 (PEG-600) was used as an $m / z$ calibration compound.

Analysis of the packaging samples (Figure 1, Steps 1 and 3) was completed by inserting the wipe containing a dried aliquot of the extract directly into the thermal desorber for $3 \mathrm{~s}$ to $5 \mathrm{~s}$, while the ground truth evidence extract (Figure 1, Step 2), was analyzed directly, without the additional extraction described in Step 4. Identification of compounds of interest in the resulting mass spectra was accomplished by searching the mass spectra against an in-house search list of over 700 compounds using Mass Mountaineer (Fineview, NY, USA). Relevant search settings included a tolerance of $\pm 0.005 \mathrm{Da}$ and a peak identification threshold of $2 \%$ relative intensity.

\section{Quantitative LC-MS/MS Analysis}

The wipe extract prepared for quantitative analysis was analyzed by LC-MS/MS using a procedure that has been described in detail elsewhere[4]. Measurements for six of the observed drugs (cocaine, fentanyl, heroin, MDMA, methamphetamine, and oxycodone) were obtained. Analysis was completed using a Thermo UltiMate 3000 liquid chromatography system (Thermo Fisher Scientific, Waltham, MA, USA) coupled to a Sciex 4000 QTrap mass spectrometer (Sciex, Framingham, MA, USA). The LC-MS/MS method used here was identical to that described previously[4] with the exception of only targeting the six drugs of interest. The resulting raw quantitative values were then adjusted to estimate the total amount of material present on the packaging using the following assumption: the collection efficiency of the wipe was approximately $33 \%$ [18] and therefore the amount of material on the packaging was approximately three-times the value obtained after doubling to account for the initial $4 \mathrm{~mL}$ extract being split into two vials for the separate analyses.

\section{Results \& Discussion}

\section{Qualitative Results}

Analysis of the outer packaging wipes taken prior to opening the evidence (Table 1) showed that detection of at least one of the drugs in the evidence extract was present on the exterior of the outer packaging roughly $80 \%$ of the time. This was significantly higher than was reported in a previous study where agreement was only $32 \%[16]$. This may have been driven by the higher fraction of cocaine cases in this dataset compared to the previous dataset. These cases typically have higher residue levels then other drugs, increasing the likelihood for detection. For both cases where no controlled substances were found in the evidence extract, the wipes of the outer packaging were also found to not contain any controlled substances. One case had only cocaine present on the exterior of the packaging, but a different drug was found in the evidence extract itself, which is not unexpected given the prevalence of cocaine in the environment[8]. Five of the six cases that had a drug present on the outer packaging that matched at least one of the drugs present in the evidence extract also had cocaine present on the exterior, however, in three of these instances, cocaine was present in an additional item submitted for that case. Finally, nine of the cases that did contain drugs in the evidence extract had no detectable levels on the exterior of the outer packaging prior to opening the case. 
Table 1. Summary of the results obtained from the qualitative analysis of the outer drug evidence packaging preand post- case analysis wipes. Note that for some cases there were multiple submissions of items from the same case which were repackaged into a single Kapak.

\begin{tabular}{|c|c|c|c|}
\hline Extract & Outer Packaging & $\begin{array}{c}\text { Pre-Opening } \\
\text { Occurrence }\end{array}$ & $\begin{array}{c}\text { Post-Repackaging } \\
\text { Occurrence }\end{array}$ \\
\hline Drug(s) Detected & Same Drug(s) Detected & $81.3 \%(n=52)$ & $93.3 \%(n=56)$ \\
\hline Drug(s) Detected & Different Drug(s) Detected & $1.6 \%(n=1)$ & $0 \%(n=0)$ \\
\hline Drug(s) Detected & No Drug Detected & $14.1 \%(n=9)$ & $5 \%(n=3)$ \\
\hline No Drugs Detected & No Drug Detected & $3.1 \%(n=2)$ & $1.6 \%(n=1)$ \\
\hline
\end{tabular}

As shown in Table 1, a substantial increase in the detection of case-related drugs was seen on the post-repackaging wipes (Row 1, increase from $81.3 \%$ to $93.3 \%$ ). All cases that had a matching drug present on the pre-opening wipe also had the same drug present on the post-repackaging wipe. As discussed in the following section, nearly all of those instances also saw increases in the amount of material present, indicating that the drug residue was not solely attributable to residue remaining on the packaging after the initial wipe prior to opening the packaging. In ten of the instances, samples that contained drugs had different or no drugs found on the pre-opening wipe $(n=1$ and $n=9$, respectively). Both of these values decreased in the post-repackaging wipes dataset $(n=0$ and $n=3$, respectively), indicating that case-specific drugs were being transferred onto the Kapaks. It should be noted that in four instances of the repackaging using new Kapak bags, multiple items from the same case that were submitted separately were combined into a single post-analysis Kapak, leading to a total of 60 post-repackaging wipes instead of 64 . There appeared to be no obvious difference in terms of the residue present after repackaging between those repackaged in the original Kapak versus those repackaged into a new Kapak. Transfer of buprenorphine residue from the analysis of suboxone strips was minimal. For both samples, no buprenorphine was present on the preopening wipes and only one post-repackaging wipe produced a low-intensity peak for buprenorphine. Supplemental Table 1 shows the full TD-DART-MS results for all 64 of the submissions.

\section{Quantitative Results}

In addition to looking at the qualitative presence or absence of drugs on the evidence packaging, quantitative information was also obtained for six of the compounds which an LC-MS/MS method existed for in-house (cocaine, fentanyl, heroin, MDMA, methamphetamine, and oxycodone). Wipes of the outer evidence packaging taken prior to opening (Figure 1, Step 1) highlighted that drug residue is present on the majority of evidence packaging prior to analysis. Out of the 62 submissions that contained one or more controlled substances (two submissions contained no controlled substances), 46 submissions $(\approx 74 \%$ ) had quantifiable levels of at least one of the drugs present in the case. The amount of material recovered was typically sub- to single microgram in quantity with average recoveries ranging from $0.06 \mu \mathrm{g}$ (oxycodone) to $1.43 \mu \mathrm{g}$ (heroin). Ten packages had initial masses of greater than $1 \mu \mathrm{g}$ of drug present - four instances each of cocaine and heroin, and two instances of methamphetamine - with a maximum of $15.60 \mu \mathrm{g}$ of heroin recovered off one package. Full quantitative data for all wipes can be found in Supplemental Table 2.

Table 2. Summary of the results obtained from the quantitative analysis of the pre-opening and post-repackaging wipes. Both overall values and values broken out by repackaging protocol are shown. The increase or decrease of each drug on a package was treated as an individual instance. Instances where a drug was present on the packaging but not actually in the case are not included in this dataset. Reported values were adjusted to incorporate a $33 \%$ collection efficiency. 


\begin{tabular}{|c|c|c|c|c|c|c|}
\hline & $\begin{array}{c}\text { \# of Times } \\
\text { Amount } \\
\text { Decreased }\end{array}$ & $\begin{array}{c}\text { \# of Times } \\
\text { Amount } \\
\text { Increased }\end{array}$ & $\begin{array}{c}\% \text { of Times } \\
\text { Amount } \\
\text { Increased }\end{array}$ & $\begin{array}{c}\text { Minimum } \\
\text { Increase }(\mu \mathrm{g})\end{array}$ & $\begin{array}{c}\text { Maximum } \\
\text { Increase }(\mu \mathrm{g})\end{array}$ & $\begin{array}{c}\text { Mean } \\
\text { Increase }(\mu \mathrm{g})\end{array}$ \\
\hline $\begin{array}{c}\text { Overall } \\
(\mathrm{n}=66)\end{array}$ & 9 & 57 & $86.4 \%$ & 0.03 & 49.47 & 4.28 \\
\hline $\begin{array}{c}\text { New Kapak } \\
(\mathrm{n}=50)\end{array}$ & 4 & 46 & $92.0 \%$ & 0.03 & 49.47 & 3.93 \\
\hline $\begin{array}{c}\text { Original } \\
\text { Kapak }(\mathrm{n}=16)\end{array}$ & 5 & 11 & $68.8 \%$ & 0.45 & 26.07 & 5.71 \\
\hline
\end{tabular}

Wipes taken after repackaging showed, on average, a two- to three-fold increase in the amount of material when compared to the pre-analysis wipes. Post-repackaging wipes had average recovered masses above $1 \mu \mathrm{g}$ for cocaine $(2.87 \mu \mathrm{g})$, heroin $(8.05 \mu \mathrm{g})$, methamphetamine $(8.72 \mu \mathrm{g})$, and oxycodone $(10.00 \mu \mathrm{g})$. As shown in Table 2 , for $86.4 \%$ of all instances (individual drugs detected in a sample) an increase in recovered mass between the preopening wipe and post-repackaging wipe was observed. The overall average increase in amount of recovered material was $4.28 \mu \mathrm{g}$, with a maximum increase of $49.47 \mu \mathrm{g}$. Only nine of the instances $(13.6 \%)$ showed a decrease in amount of material - which would be attributed to either use of new Kapak that did not have residue transferred on it or a reduction in mass in the post-repackaging wipe due to the removal of residue with initial pre-opening wipe. Surprisingly, repackaging of evidence into a new Kapak did not lead to a reduction in residue on the packaging exterior as may have been expected (Table 2). Potential reasons for this observation are discussed in detail below. The samples repackaged into a new Kapak had a slightly higher percentage of samples that saw an increase in drug amounts, while $69 \%$ of the samples that were repackaged into the original Kapak showed an increase. This observation may be due to the smaller sample size for the original Kapak samples ( $n=16$ compared to $n=50$ ) or may be due to variations in analysis protocols at both laboratories.

\section{Use of Inner Packaging Residue to Predict Contents}

Since wiping of the outer evidence packaging was being completed for the study, it was decided that the inner packaging (bag, bottle, street packaging, etc.) would also be wiped to determine if the ability to accurately predict the contents based on the residue aligned with previously published work[16]. As with the outer packaging wipes, the inner packaging wipes were analyzed both qualitatively and quantitatively and a positive result was defined as the presence of at least one of the drugs in the case present in the residue on the exterior of the inner packaging. While the full results can be found in Supplemental Table 3 (qualitative, TD-DART-MS) and Supplemental Table 4 (quantitative, LC-MS/MS), summary results are presented in Tables 3 and 4 . In total,74 of the 82 inner packages that were wiped had at least one drug present that agreed with the evidence contents ( $90.2 \%$ of samples), and the two samples that contained no controlled substances within the evidence sample had no drug residues on them (2.4\% of samples), leading to an overall accuracy of $92.7 \%$. This value is almost identical to previous work which found an overall accuracy of $92 \%$, and had just over twice the population size $(n=191)[16]$. Six false negatives were found within the samples - instances where a drug was present in the evidence extract, but no residue was detected on the wipe using TD-DART-MS with two of those instances being the suboxone strips, one methamphetamine submission, two heroin submissions, and one heroin and fentanyl submission. Additionally, as expected, and as shown in previous work[16], the presence of cocaine on many of the inner packaging wipes was found even if cocaine was not present in the case - which may be attributable to environmental background from where the evidence was seized. 
Table 3. Summary of the results obtained from the qualitative analysis of the inner packaging.

\begin{tabular}{|c|c|c|c|}
\hline Extract & Inner Packaging & Result Type & Occurrence \\
\hline Drug(s) Detected & Same Drug(s) Detected & True Positive & $90.2 \%(\mathrm{n}=74)$ \\
\hline Drug(s) Detected & Different Drug(s) Detected & False Positive & $0 \%(\mathrm{n}=0)$ \\
\hline No Drug Detected & Drug(s) Detected & False Positive & $0 \%(\mathrm{n}=0)$ \\
\hline Drug(s) Detected & No Drug Detected & False Negative & $7.3 \%(\mathrm{n}=6)$ \\
\hline No Drug Detected & No Drug Detected & True Negative & $2.4 \%(\mathrm{n}=2)$ \\
\hline & & Overall Accuracy: & $\mathbf{9 2 . 7} \%$ \\
\hline
\end{tabular}

As with the qualitative results, the quantitative results (Table 4) also showed good agreement with previous findings. Average amounts of material, after adjusting for the $33 \%$ collection efficiency[18], ranged from single to tens of micrograms per sample. Heroin, as with previous work[16], had the highest amounts of material, with a maximum amount of over half a milligram present on one of the inner packages. Given the fact that fentanyl is typically heavily cut in street samples, it was expected that it would have the lowest amount, on average, though amounts as high as $33 \mu \mathrm{g}$ were found. These results, compounded with previous findings, highlight the need for cautious handling of evidence to prevent potential unintentional exposure through residues.

Table 4. Summary of the results obtained from the quantitative analysis of the inner drug evidence packaging. The values reported here assume that the collection efficiency of the meta-aramid wipe was $33 \%$.

\begin{tabular}{|c|c|c|c|c|}
\hline & $\begin{array}{c}\text { \# of } \\
\text { Samples }\end{array}$ & $\begin{array}{c}\text { Average Amount on } \\
\text { Packaging }(\boldsymbol{\mu g})\end{array}$ & $\begin{array}{c}\text { Minimum Amount on } \\
\text { Packaging }(\boldsymbol{\mu g})\end{array}$ & $\begin{array}{c}\text { Maximum Amount on } \\
\text { Packaging }(\boldsymbol{\mu} \mathbf{g})\end{array}$ \\
\hline Cocaine & 43 & 1.93 & 0.03 & 19.53 \\
\hline Fentanyl & 21 & 2.59 & 0.03 & 32.88 \\
\hline Heroin & 24 & 46.96 & 0.12 & 591.00 \\
\hline MDMA & 1 & 4.95 & $\mathrm{~N} / \mathrm{A}$ & $\mathrm{N} / \mathrm{A}$ \\
\hline Methamphetamine & 5 & 11.46 & 0.24 & 28.00 \\
\hline Oxycodone & 1 & 18.57 & $\mathrm{~N} / \mathrm{A}$ & $\mathrm{N} / \mathrm{A}$ \\
\hline
\end{tabular}

\section{Conclusions}

The most important conclusion drawn from these studies was that the process of opening, analyzing, and repackaging drug evidence led to an increase in residue on the outer packaging. This increase was likely unavoidable given the routine analysis process. The growing body of research on drug background and the processes that contribute to it highlights the inherent risks of handling dangerous powders. Simply opening a plastic or wax-paper bag containing powder will release particulate into the air, allowing it to settle on nearby surfaces. Instead of trying to eliminate the presence of residue on evidence packaging, a more practical goal would be to identify the practices that tend to contribute to or prevent the accumulation of residue and develop a best practice aimed at achieving the lowest levels reasonably obtainable. Identifying these practices are the focus of ongoing work and include identifying when, and where, to store the original or new Kapak bags, whether changing gloves prior to re-packaging would lower residue levels, and what other cleaning and handling practices could reduce residue levels. Alternatively, one could look at ways to clean packaging after analysis, but the identified approaches would need to be rapid and not interfere with items such as signatures, markings, and evidence tape that may be present. 
The results of this work also highlight the need for situational awareness beyond drug chemists. Evidence handling technicians, crime scene technicians, submitting officers, and those further down the evidence handling chain should also be aware that there is a high probability the packaging they are handling is contaminated with drug residue. This type of awareness may prompt increased used of personal protective equipment, namely gloves, to prevent accidental exposure. Residue present in storage containers, which can accumulate from transfer of residue off evidence packaging is another area where increased awareness is likely beneficial. There are also potential questions that could arise with short term and long-term storage of these materials as well.

Overall, this work demonstrated that trace drug residues can be transferred onto the outer evidence packaging during the case analysis process. There was no clear benefit in repackaging of evidence in the original Kapak bag or a new Kapak, though this may be a function of how, when, and where evidence gets repackaged. Levels of controlled substances on the exterior of the outer bags typically were in the sub-microgram to single microgram range, though tens of micrograms were found in some instances. Collection of residues from the inner packaging showed good agreement with previous work and again highlight the potential to leverage trace residue for presumptive screening, especially for the presence of opioids for triaging of evidence. While current efforts are focusing on identifying best practices to minimize the transfer of drug residue to evidence packaging, this work provides another piece to the growing body of drug background research.

\section{Disclaimer}

Certain commercial products are identified in order to adequately specify the procedure; this does not imply endorsement or recommendation by NIST, nor does it imply that such products are necessarily the best available for the purpose.

Certain commercial products are identified in order to adequately specify the procedure; this does not imply endorsement or recommendation by Maryland State Police, nor does it imply that such products are necessarily the best available for the purpose.

Certain commercial products are identified in order to adequately specify the procedure; this does not imply endorsement or recommendation by Vermont Forensic Laboratory, nor does it imply that such products are necessarily the best available for the purpose.

\section{References}

[1] Kendra R. Broadwater, David A. Jackson, Jessica F. Li, Evaluation of occupational exposures to illicit drugs at controlled substances laboratories., U.S. Department of Health and Human Services, Public Health Service, Centers for Disease Control and Prevention, National Institute for Occupational Safety and Health, 2020. https://doi.org/10.26616/NIOSHHHE201800903366.

[2] CDC - Fentanyl: Emergency Responders at Risk - NIOSH Workplace Safety \& Health Topics, (2020). https://www.cdc.gov/niosh/topics/fentanyl/risk.html (accessed March 25, 2020).

[3] J.L. Burgess, S. Barnhart, H. Checkoway, Investigating clandestine drug laboratories: Adverse medical effects in law enforcement personnel, American Journal of Industrial Medicine. 30 (n.d.) 488-494. https://doi.org/10.1002/(SICI)1097-0274(199610)30:4<488::AID-AJIM15>3.0.CO;2-0.

[4] E. Sisco, M. Najarro, A. Burns, A snapshot of drug background levels on surfaces in a forensic laboratory, Forensic Chemistry. 11 (2018) 47-57. https://doi.org/10.1016/j.forc.2018.09.001.

[5] E. Sisco, M. Najarro, A multi-laboratory investigation of drug background levels, Forensic Chemistry. 16 (2019) 100184. https://doi.org/10.1016/j.forc.2019.100184.

[6] S. Armenta, M. de la Guardia, M. Alcalà, M. Blanco, C. Perez-Alfonso, N. Galipienso, Ion mobility spectrometry evaluation of cocaine occupational exposure in forensic laboratories, Talanta. 130 (2014) 251258. https://doi.org/10.1016/j.talanta.2014.06.044. 
[7] G.S. Doran, R.M. Deans, C.D. Filippis, C. Kostakis, J.A. Howitt, Quantification of licit and illicit drugs on typical police station work surfaces using LC-MS/MS, Anal. Methods. 9 (2017) 198-210. https://doi.org/10.1039/C6AY02668K.

[8] G.S. Doran, R. Deans, C. De Filippis, C. Kostakis, J.A. Howitt, The presence of licit and illicit drugs in police stations and their implications for workplace drug testing, Forensic Sci. Int. 278 (2017) 125-136. https://doi.org/10.1016/j.forsciint.2017.06.034.

[9] E. Sisco, M.E. Staymates, A. Burns, An easy to implement approach for laboratories to visualize particle spread during the handling and analysis of drug evidence, Forensic Chemistry. 18 (2020) 100232. https://doi.org/10.1016/j.forc.2020.100232.

[10] E. Sisco, M.E. Staymates, L.M. Watt, Net weights: Visualizing and quantifying their contribution to drug background levels in forensic laboratories, Forensic Chemistry. 20 (2020) 100259. https://doi.org/10.1016/j.forc.2020.100259.

[11] P.J.M. Sessink, N.S.S. Friemèl, R.B.M. Anzion, R.P. Bos, Biological and environmental monitoring of occupational exposure of pharmaceutical plant workers to methotrexate, Int Arch Occup Environ Health. 65 (1994) 401-403. https://doi.org/10.1007/BF00383251.

[12] H. Nakashizuka, J. Shoji, H. Shimada, M. Yuzawa, EXPERIMENTAL VISUALIZATION AND QUANTIFICATION OF VITREOUS CONTAMINATION FOLLOWING INTRAVITREAL INJECTIONS, (2016). https://doi.org/info:doi/10.1097/IAE.0000000000001028.

[13] L. Casanova, E. Alfano-Sobsey, W.A. Rutala, D.J. Weber, M. Sobsey, Virus Transfer from Personal Protective Equipment to Healthcare Employees' Skin and Clothing, Emerging Infectious Diseases. 14 (n.d.). https://doi.org/10.3201/eid1408.080085.

[14] M.E. Tomas, S. Kundrapu, P. Thota, V.C.K. Sunkesula, J.L. Cadnum, T.S.C. Mana, A. Jencson, M. O'Donnell, T.F. Zabarsky, M.T. Hecker, A.J. Ray, B.M. Wilson, C.J. Donskey, Contamination of Health Care Personnel During Removal of Personal Protective Equipment, JAMA Intern Med. 175 (2015) 1904-1910. https://doi.org/10.1001/jamainternmed.2015.4535.

[15] J. Maitland, R. Boyer, D. Gallagher, S. Duncan, N. Bauer, J. Kause, J. Eifert, Tracking Cross-Contamination Transfer Dynamics at a Mock Retail Deli Market Using GloGerm, Journal of Food Protection. 76 (2013) 272282. https://doi.org/10.4315/0362-028X.JFP-12-271.

[16] E. Sisco, E.L. Robinson, A. Burns, R. Mead, What's in the bag? Analysis of exterior drug packaging by TDDART-MS to predict the contents, Forensic Science International. 304 (2019) 109939. https://doi.org/10.1016/j.forsciint.2019.109939.

[17] E. Sisco, T.P. Forbes, M.E. Staymates, G. Gillen, Rapid analysis of trace drugs and metabolites using a thermal desorption DART-MS configuration, Anal. Methods. 8 (2016) 6494-6499. https://doi.org/10.1039/C6AY01851C.

[18] E. L. Robinson, E. Sisco, M. E. Staymates, J. A. Lawrence, A new wipe-sampling instrument for measuring the collection efficiency of trace explosives residues, Analytical Methods. 10 (2018) 204-213. https://doi.org/10.1039/C7AY02694C. 


\section{Supplemental Information}

Table S1. Qualitative TD-DART-MS results for the wipes obtained from the outer packaging prior to opening and after repackaging. Note that items from the same case share a post-repackaging result as they were re-packaged together.

\begin{tabular}{|c|c|c|c|c|}
\hline Case \# & Repackaging & Contents & $\begin{array}{c}\text { TD-DART-MS Result (Pre- } \\
\text { Opening Wipe) }\end{array}$ & $\begin{array}{c}\text { TD-DART-MS Result (Post- } \\
\text { Repackaging Wipe) }\end{array}$ \\
\hline 1 & New & Cocaine & Cocaine & Cocaine \\
\hline 2 & New & Fentanyl, Heroin & No Drugs Detected & Fentanyl, Heroin \\
\hline 3 & New & Cocaine & Cocaine & Cocaine \\
\hline 4 & New & Cocaine & Cocaine & Cocaine \\
\hline \multirow{2}{*}{5} & New & Cocaine, Heroin, 4-ANPP & Cocaine & \multirow{2}{*}{ Cocaine, Heroin } \\
\hline & New & Cocaine & Cocaine & \\
\hline 6 & New & Fentanyl, Heroin, 4-ANPP & No Drugs Detected & Heroin \\
\hline 7 & New & Heroin & Heroin & Heroin \\
\hline 8 & New & Cocaine, Fentanyl, Heroin & Cocaine, Heroin & Fentanyl, Heroin \\
\hline 9 & New & Fentanyl, 4-ANPP & No Drugs Detected & No Drugs Detected \\
\hline 10 & New & Cocaine & Cocaine & Cocaine \\
\hline \multirow{3}{*}{11} & New & Heroin & Cocaine, Heroin & \multirow{3}{*}{ Cocaine, Heroin } \\
\hline & New & Cocaine & Cocaine & \\
\hline & New & No Controlled Substances & No Drugs Detected & \\
\hline \multirow[b]{2}{*}{12} & New & Cocaine & Cocaine & \multirow{2}{*}{ Cocaine, Fentanyl } \\
\hline & New & Fentanyl & Cocaine, Fentanyl & \\
\hline \multirow[t]{2}{*}{13} & New & $\begin{array}{c}\text { Cocaine, Fentanyl, Heroin, 4- } \\
\text { ANPP }\end{array}$ & Cocaine, Fentanyl, Heroin & \multirow[t]{2}{*}{ Cocaine, Fentanyl, Heroin } \\
\hline & New & Heroin & Cocaine, Fentanyl, Heroin & \\
\hline 14 & New & Cocaine & Cocaine & Cocaine \\
\hline 15 & New & Cocaine & Cocaine & Cocaine \\
\hline 16 & New & Cocaine & Cocaine & Cocaine \\
\hline 17 & New & Methamphetamine & No Drugs Detected & No Drugs Detected \\
\hline 18 & New & Methamphetamine & Methamphetamine & Methamphetamine \\
\hline 19 & New & Fentanyl, Heroin & No Drugs Detected & Fentanyl, Heroin \\
\hline 20 & New & Fentanyl, Heroin, Tramadol & Heroin & Fentanyl, Heroin, Tramadol \\
\hline 21 & New & Fentanyl, Heroin, Tramadol & Heroin & Fentanyl, Heroin, Tramadol \\
\hline 22 & New & MDMA & MDMA & MDMA \\
\hline 23 & New & Heroin & Heroin & Heroin \\
\hline 24 & New & Fentanyl, Heroin & Fentanyl, Heroin & Fentanyl, Heroin \\
\hline 25 & New & $\begin{array}{l}\text { Heroin, Fentanyl, 4-ANPP, } \\
\text { Codeine, Ketamine }\end{array}$ & Heroin & Fentanyl, Heroin \\
\hline 26 & New & Heroin & Heroin & Heroin \\
\hline 27 & New & Fentanyl, Heroin, 4-ANPP & Heroin & Fentanyl, Heroin \\
\hline 28 & New & Cocaine & Cocaine & Cocaine \\
\hline 29 & New & Methamphetamine & Methamphetamine & Methamphetamine \\
\hline 30 & New & Cocaine & Cocaine & Cocaine \\
\hline 31 & New & Cocaine & Cocaine & Cocaine \\
\hline 32 & New & Cocaine & Cocaine & Cocaine \\
\hline 33 & New & Fentanyl, 4-ANPP & No Drugs Detected & Fentanyl \\
\hline 34 & New & Methamphetamine & Methamphetamine & Methamphetamine \\
\hline 35 & New & No Controlled Substances & No Drugs Detected & No Drugs Detected \\
\hline 36 & New & Cocaine & Cocaine & Cocaine \\
\hline 37 & New & Cocaine & Cocaine & Cocaine \\
\hline 38 & New & Cocaine & No Drugs Detected & Cocaine \\
\hline 39 & New & Cocaine & Cocaine & Cocaine \\
\hline 40 & New & Cocaine & Cocaine & Cocaine \\
\hline 41 & New & Cocaine & Cocaine & Cocaine \\
\hline 42 & Original & Cocaine & Cocaine & Cocaine \\
\hline 43 & Original & Cocaine & Cocaine & Cocaine \\
\hline 44 & Original & Cocaine & Cocaine & Cocaine \\
\hline 45 & Original & Eutylone & Eutylone & Eutylone, Cocaine \\
\hline 46 & Original & Fentanyl, Heroin, 4-ANPP & Fentanyl & Fentanyl \\
\hline 47 & Original & Cocaine & Cocaine & Cocaine \\
\hline 48 & Original & Fentanyl, 4-ANPP & Cocaine & Cocaine, Fentanyl \\
\hline 49 & Original & Cocaine & Cocaine & Cocaine \\
\hline 50 & Original & Buprenorphine & No Drugs Detected & No Drugs Detected \\
\hline 51 & Original & Oxycodone & Oxycodone & Oxycodone \\
\hline 52 & Original & Cocaine & Cocaine & Cocaine \\
\hline 53 & Original & Cocaine & Cocaine & Cocaine \\
\hline 54 & Original & Cocaine, Fentanyl, 4-ANPP & Cocaine, Fentanyl & Cocaine, Fentanyl \\
\hline 55 & Original & Buprenorphine & No Drugs Detected & Buprenorphine \\
\hline
\end{tabular}




\begin{tabular}{|c|c|c|c|c|}
\hline 56 & Original & Cocaine, Fentanyl & Cocaine, Fentanyl & Cocaine, Fentanyl \\
\hline 57 & Original & Methamphetamine, MDMA & Cocaine, Methamphetamine & $\begin{array}{c}\text { Cocaine, Methamphetamine, } \\
\text { MDMA }\end{array}$ \\
\hline 58 & Original & Cocaine & Cocaine, Methamphetamine & Cocaine \\
\hline 59 & Original & $\begin{array}{c}\text { Fentanyl, Heroin, 4-ANPP, } \\
\text { Etizolam, Deschloroetizolam }\end{array}$ & Cocaine, Fentanyl & Cocaine, Fentanyl, Etizolam \\
\hline
\end{tabular}


Table S2. Quantitative LC-MS/MS results for the wipes obtained from the outer packaging prior to opening and after repackaging. Abbreviations for the drugs include $C=$ cocaine, $F=$ fentanyl, $H=$ heroin, $D=M D M A, M=$ methamphetamine, and $\mathrm{O}=$ oxycodone. The values presented here include the assumed $33 \%$ collection efficiency for the wipes. Note that items from the same case share a post-repackaging result as they were re-packaged together.

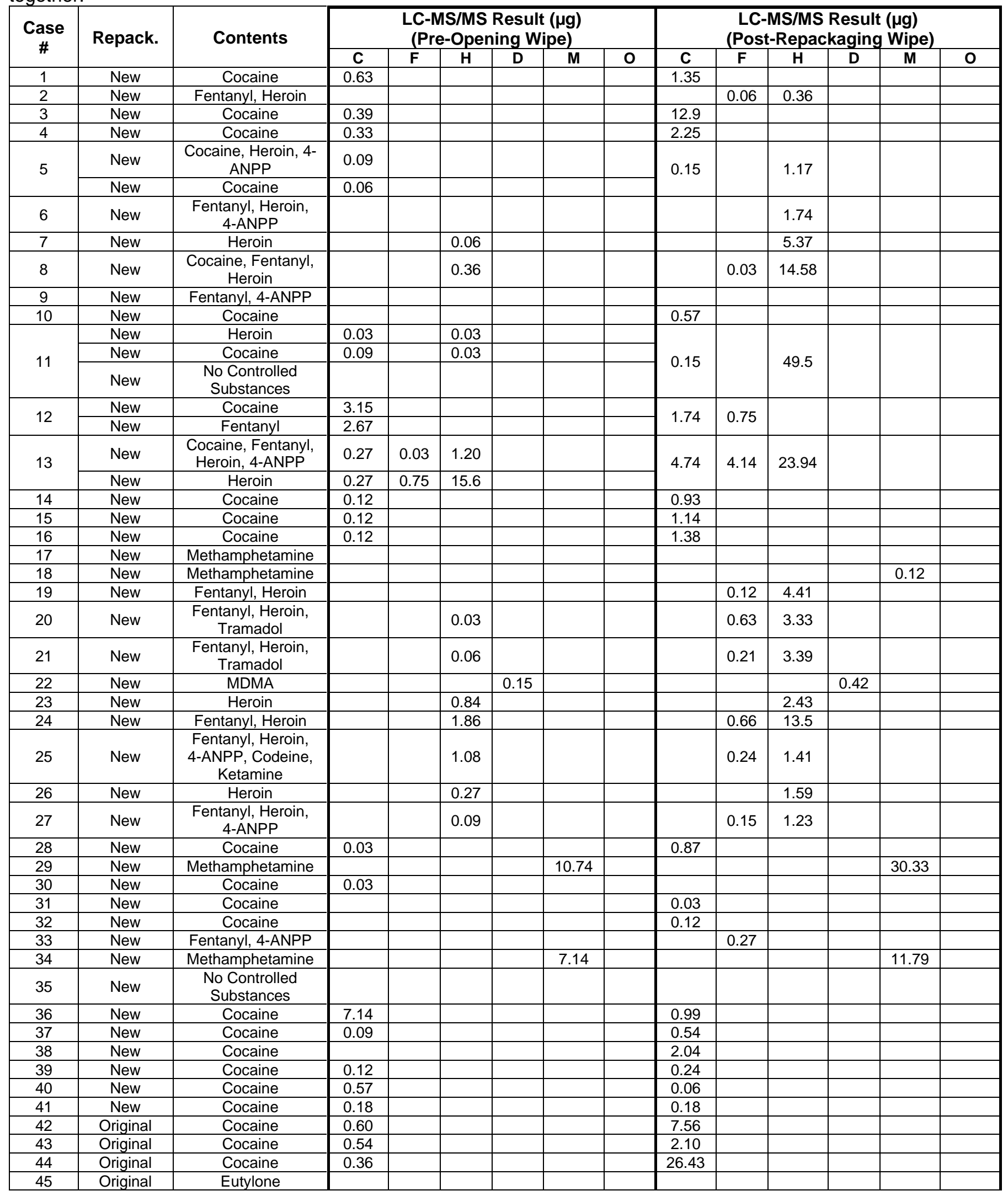




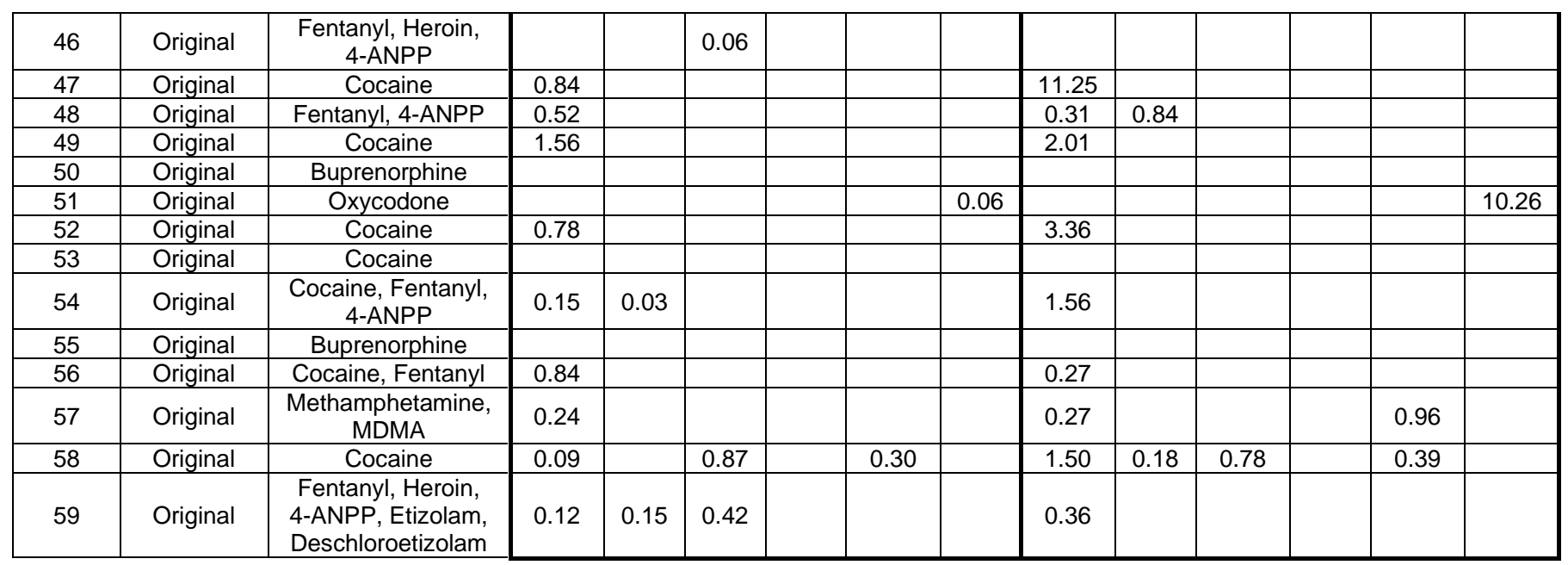


Table S3. Qualitative TD-DART-MS results from the inner packaging wipes.

\begin{tabular}{|c|c|c|c|c|}
\hline Case \# & Item \# & $\begin{array}{l}\text { Packaging } \\
\text { Type }\end{array}$ & Contents & TD-DART-MS Result \\
\hline 1 & 1 & Plastic & Cocaine & Cocaine \\
\hline \multirow{2}{*}{2} & 1 & Glassine & Heroin & Cocaine \\
\hline & 2 & Glassine & Fentanyl, Heroin & Fentanyl \\
\hline 3 & 1 & Plastic & Cocaine & Cocaine \\
\hline 4 & 1 & Plastic & Cocaine & Cocaine \\
\hline \multirow{2}{*}{5} & 1 & Plastic & Cocaine, Heroin, 4-ANPP & Cocaine \\
\hline & 2 & Plastic & Cocaine & Cocaine \\
\hline 6 & 1 & Glassine & Fentanyl, Heroin, 4-ANPP & Fentanyl, Heroin \\
\hline \multirow{2}{*}{7} & 1 & Plastic & Heroin & No Drugs Detected \\
\hline & 2 & Glassine & Heroin & Heroin \\
\hline 8 & 1 & Foil & Cocaine, Fentanyl, Heroin & Fentanyl, Heroin \\
\hline 9 & 1 & Plastic & Fentanyl, 4-ANPP & Fentanyl \\
\hline 10 & 1 & Plastic & Cocaine & Cocaine \\
\hline \multirow{3}{*}{11} & 1 & Plastic & Heroin & Cocaine, Heroin \\
\hline & 1 & Plastic & Cocaine & Cocaine \\
\hline & 1 & Plastic & No Controlled Substances & No Drugs Detected \\
\hline \multirow{2}{*}{12} & 1 & Plastic & Cocaine & Cocaine \\
\hline & 1 & Glassine & Fentanyl & Cocaine, Fentanyl \\
\hline \multirow[t]{2}{*}{13} & 1 & Plastic & $\begin{array}{c}\text { Cocaine, Fentanyl, Heroin, 4- } \\
\text { ANPP }\end{array}$ & Cocaine, Fentanyl, Heroin \\
\hline & 1 & Glassine & Heroin & Cocaine, Fentanyl, Heroin \\
\hline 14 & 1 & Plastic & Cocaine & Cocaine \\
\hline 15 & 1 & Plastic & Cocaine & Cocaine \\
\hline 16 & 1 & Plastic & Cocaine & Cocaine \\
\hline 17 & 1 & Plastic & Methamphetamine & No Drugs Detected \\
\hline 18 & 1 & Plastic & Methamphetamine & Methamphetamine \\
\hline \multirow{2}{*}{19} & 1 & Glassine & Fentanyl, Heroin & Fentanyl, Heroin \\
\hline & 2 & Glassine & Fentanyl, Heroin & Fentanyl, Heroin \\
\hline \multirow{2}{*}{20} & 1 & Glassine & Fentanyl, Heroin, Tramadol & Fentanyl, Heroin, Tramadol \\
\hline & 2 & Glassine & Fentanyl, Heroin, Tramadol & Fentanyl, Heroin, Tramadol \\
\hline \multirow[b]{2}{*}{21} & 1 & Glassine & Fentanyl, Heroin, Tramadol & Fentanyl, Heroin, Tramadol \\
\hline & 2 & Glassine & Fentanyl, Heroin, Tramadol & Fentanyl, Heroin, Tramadol \\
\hline 22 & 1 & Plastic & MDMA & MDMA \\
\hline \multirow{2}{*}{23} & 1 & Glassine & Heroin & Heroin \\
\hline & 2 & Glassine & Heroin & Heroin \\
\hline \multirow[b]{2}{*}{24} & 1 & Glassine & Fentanyl, Heroin & Fentanyl, Heroin \\
\hline & 2 & Glassine & Fentanyl, Heroin & Fentanyl, Heroin \\
\hline \multirow{2}{*}{25} & 1 & Glassine & $\begin{array}{l}\text { Fentanyl, Heroin, 4-ANPP, } \\
\text { Codeine, Ketamine }\end{array}$ & Fentanyl, Heroin \\
\hline & 2 & Glassine & $\begin{array}{l}\text { Fentanyl, Heroin, 4-ANPP, } \\
\text { Codeine }\end{array}$ & Fentanyl, Heroin \\
\hline \multirow{2}{*}{26} & 1 & Glassine & Heroin & Heroin \\
\hline & 2 & Plastic & Heroin & Heroin \\
\hline \multirow{2}{*}{27} & 1 & Glassine & Fentanyl, Heroin, 4-ANPP & Fentanyl \\
\hline & 2 & Glassine & Fentanyl, Heroin, 4-ANPP & Fentanyl \\
\hline 28 & 1 & Foil & Cocaine & Cocaine \\
\hline 29 & 1 & Plastic & Methamphetamine & Methamphetamine \\
\hline 30 & 1 & Plastic & Cocaine & Cocaine \\
\hline 31 & 1 & Plastic & Cocaine & Cocaine \\
\hline 32 & 1 & Plastic & Cocaine & Cocaine \\
\hline 33 & 1 & Glassine & Fentanyl, 4-ANPP & Fentanyl \\
\hline 33 & 2 & Glassine & Fentanyl, 4-ANPP & Fentanyl, 4-ANPP \\
\hline 34 & 1 & Plastic & Methamphetamine & Methamphetamine \\
\hline 35 & 1 & Other & No Controlled Substances & No Drugs Detected \\
\hline 36 & 1 & Plastic & Cocaine & Cocaine \\
\hline 37 & 1 & Plastic & Cocaine & Cocaine \\
\hline 38 & 1 & Plastic & Cocaine & Cocaine \\
\hline 39 & 1 & Paper & Cocaine & Cocaine \\
\hline 40 & 1 & Plastic & Cocaine & Cocaine \\
\hline 41 & 1 & Plastic & Cocaine & Cocaine \\
\hline 42 & 1 & Plastic & Cocaine & Cocaine \\
\hline 42 & 2 & Plastic & Cocaine & Cocaine \\
\hline 43 & 1 & Plastic & Cocaine & Cocaine \\
\hline 44 & 1 & Plastic & Cocaine & Cocaine \\
\hline 45 & 1 & Plastic & Eutylone & Eutylone, Cocaine \\
\hline 46 & 1 & Plastic & Fentanyl, Heroin, 4-ANPP & No Drugs Detected \\
\hline 47 & 1 & Plastic & Cocaine & Cocaine \\
\hline
\end{tabular}




\begin{tabular}{|c|c|c|c|c|}
\hline 48 & 1 & Plastic & Fentanyl, 4-ANPP & Fentanyl \\
\hline 49 & 1 & Plastic & Cocaine & Cocaine \\
\hline 50 & 1 & $\begin{array}{c}\text { Suboxone } \\
\text { Packet }\end{array}$ & Buprenorphine & No Drugs Detected \\
\hline 51 & 1 & Currency & Oxycodone & Oxycodone \\
\hline 52 & 1 & Plastic & Cocaine & Cocaine \\
\hline 53 & 1 & Plastic & Cocaine & Cocaine \\
\hline \multirow{2}{*}{54} & 1 & Plastic & Cocaine, Fentanyl, 4-ANPP & Cocaine \\
\hline & 2 & Plastic & Cocaine, Fentanyl, 4-ANPP & Cocaine \\
\hline 55 & 1 & Plastic & Buprenorphine & No Drugs Detected \\
\hline 56 & 1 & Plastic & Cocaine, Fentanyl & Cocaine, Fentanyl \\
\hline \multirow{3}{*}{57} & 1 & Plastic & Methamphetamine & Cocaine, Methamphetamine \\
\hline & 2 & Plastic & Methamphetamine & Cocaine, Methamphetamine \\
\hline & 3 & Plastic & MDMA & Cocaine, MDMA \\
\hline \multirow{3}{*}{58} & 1 & Plastic & Cocaine & Cocaine \\
\hline & 2 & Plastic & Cocaine & Cocaine \\
\hline & 3 & Plastic & Cocaine & Cocaine \\
\hline \multirow{2}{*}{59} & 1 & Plastic & $\begin{array}{l}\text { Fentanyl, Heroin, 4-ANPP, } \\
\text { Etizolam, Deschloroetizolam }\end{array}$ & Cocaine, Fentanyl \\
\hline & 2 & Plastic & $\begin{array}{c}\text { Fentanyl, Heroin, 4-ANPP, } \\
\text { Etizolam }\end{array}$ & Cocaine, Fentanyl, Etizolam \\
\hline
\end{tabular}


Table S4. Quantitative LC-MS/MS results for wipes obtained from the inner packaging. Abbreviations for the drugs include $\mathrm{C}=$ cocaine, $\mathrm{F}=$ fentanyl, $\mathrm{H}=$ heroin, $\mathrm{D}=\mathrm{MDMA}, \mathrm{M}=$ methamphetamine, and $\mathrm{O}=$ oxycodone. The values presented here have been adjusted from the raw values based on the assumptions explained in the text.

\begin{tabular}{|c|c|c|c|c|c|c|c|c|c|}
\hline \multirow{2}{*}{ Case \# } & \multirow{2}{*}{$\begin{array}{c}\text { Item } \\
\#\end{array}$} & \multirow{2}{*}{$\begin{array}{l}\text { Packaging } \\
\text { Type }\end{array}$} & \multirow{2}{*}{ Contents } & \multicolumn{6}{|c|}{ LC-MS/MS Result $(\mu \mathrm{g})$} \\
\hline & & & & $\mathbf{C}$ & $\mathbf{F}$ & $\mathbf{H}$ & D & $\mathbf{M}$ & 0 \\
\hline 1 & 1 & Plastic & Cocaine & 2.46 & & & & & \\
\hline \multirow{2}{*}{2} & 1 & Glassine & Heroin & & 0.24 & & & & \\
\hline & 2 & Glassine & Fentanyl, Heroin & & & & & & \\
\hline 3 & 1 & Plastic & Cocaine & 19.53 & & & & & \\
\hline 4 & 1 & Plastic & Cocaine & 0.54 & & & & & \\
\hline \multirow{2}{*}{5} & 1 & Plastic & Cocaine, Heroin, 4-ANPP & 0.15 & & & & & \\
\hline & 1 & Plastic & Cocaine & 8.16 & & & & & \\
\hline 6 & 1 & Glassine & Fentanyl, Heroin, 4-ANPP & & & 0.66 & & & \\
\hline \multirow{2}{*}{7} & 1 & Plastic & Heroin & & & & & & \\
\hline & 2 & Glassine & Heroin & & & 0.12 & & & \\
\hline 8 & 1 & Foil & Cocaine, Fentanyl, Heroin & & 1.62 & 211.00 & & & \\
\hline 9 & 1 & Plastic & Fentanyl, 4-ANPP & & & & & & \\
\hline 10 & 1 & Plastic & Cocaine & 0.87 & & & & & \\
\hline \multirow{3}{*}{11} & 1 & Plastic & Heroin & 0.21 & & 3.87 & & & \\
\hline & 2 & Plastic & Cocaine & 0.12 & & & & & \\
\hline & 3 & Plastic & No Controlled Substances & & & & & & \\
\hline \multirow{2}{*}{12} & 1 & Plastic & Cocaine & 4.23 & & & & & \\
\hline & 2 & Glassine & Fentanyl & 0.60 & & & & & \\
\hline \multirow[t]{2}{*}{13} & 1 & Plastic & $\begin{array}{c}\text { Cocaine, Fentanyl, Heroin, 4- } \\
\text { ANPP }\end{array}$ & 0.15 & 32.90 & 591.00 & & & \\
\hline & 2 & Glassine & Heroin & 1.08 & 2.46 & 36.30 & & & \\
\hline 14 & 1 & Plastic & Cocaine & 3.36 & & & & & \\
\hline 15 & 1 & Plastic & Cocaine & 0.57 & & & & & \\
\hline 16 & 1 & Plastic & Cocaine & 5.34 & & & & & \\
\hline 17 & 1 & Plastic & Methamphetamine & & & & & & \\
\hline 18 & 1 & Plastic & Methamphetamine & & & & & 0.24 & \\
\hline \multirow{2}{*}{19} & 1 & Glassine & Fentanyl, Heroin & & & 0.24 & & & \\
\hline & 2 & Glassine & Fentanyl, Heroin & & 0.75 & 10.40 & & & \\
\hline 2 & 1 & Glassine & Fentanyl, Heroin, Tramadol & & 1.29 & 9.18 & & & \\
\hline$<0$ & 2 & Glassine & Fentanyl, Heroin, Tramadol & & 0.63 & 4.14 & & & \\
\hline 21 & 1 & Glassine & Fentanyl, Heroin, Tramadol & & 0.57 & 9.33 & & & \\
\hline 21 & 2 & Glassine & Fentanyl, Heroin, Tramadol & & 0.18 & 1.92 & & & \\
\hline 22 & 1 & Plastic & MDMA & & & & 4.95 & & \\
\hline 23 & 1 & Glassine & Heroin & & & 6.87 & & & \\
\hline 20 & 2 & Glassine & Heroin & & & 1.41 & & & \\
\hline 24 & 1 & Glassine & Fentanyl, Heroin & & 4.05 & 172.00 & & & \\
\hline 24 & 2 & Glassine & Fentanyl, Heroin & & 1.20 & 55.20 & & & \\
\hline 25 & 1 & Glassine & $\begin{array}{l}\text { Fentanyl, Heroin, 4-ANPP, } \\
\text { Codeine, Ketamine }\end{array}$ & & 2.49 & 1.83 & & & \\
\hline $2 v$ & 2 & Glassine & $\begin{array}{l}\text { Fentanyl, Heroin, 4-ANPP, } \\
\text { Codeine }\end{array}$ & & 0.33 & 0.45 & & & \\
\hline 26 & 1 & Glassine & Heroin & & & 0.75 & & & \\
\hline 20 & 2 & Plastic & Heroin & & & 1.08 & & & \\
\hline 27 & 1 & Glassine & Fentanyl, Heroin, 4-ANPP & & 0.15 & & & & \\
\hline 27 & 2 & Glassine & Fentanyl, Heroin, 4-ANPP & & 0.30 & & & & \\
\hline 28 & 1 & Foil & Cocaine & 1.08 & & & & & \\
\hline 29 & 1 & Plastic & Methamphetamine & & & & & 27.39 & \\
\hline 30 & 1 & Plastic & Cocaine & 0.09 & & & & & \\
\hline 31 & 1 & Plastic & Cocaine & 0.33 & & & & & \\
\hline 32 & 1 & Plastic & Cocaine & 0.30 & & & & & \\
\hline 33 & 1 & Glassine & Fentanyl, 4-ANPP & & 2.34 & & & & \\
\hline 33 & 2 & Glassine & Fentanyl, 4-ANPP & & 1.23 & & & & \\
\hline 34 & 1 & Plastic & Methamphetamine & & & & & 27.90 & \\
\hline 35 & 1 & Other & No Controlled Substances & & & & & & \\
\hline 36 & 1 & Plastic & Cocaine & 2.31 & & & & & \\
\hline 37 & 1 & Plastic & Cocaine & 0.60 & & & & & \\
\hline 38 & 1 & Plastic & Cocaine & 0.18 & & & & & \\
\hline 39 & 1 & Paper & Cocaine & 0.45 & & & & & \\
\hline 40 & 1 & Plastic & Cocaine & 0.60 & & & & & \\
\hline 41 & 1 & Plastic & Cocaine & 2.97 & & & & & \\
\hline 42 & 1 & Plastic & Cocaine & 8.85 & & & & & \\
\hline 42 & 2 & Plastic & Cocaine & 1.35 & & & & & \\
\hline 43 & 1 & Plastic & Cocaine & 1.59 & & & & & \\
\hline 44 & 1 & Plastic & Cocaine & 2.37 & & & & & \\
\hline 45 & 1 & Plastic & Eutylone & & & & & & \\
\hline
\end{tabular}




\begin{tabular}{|c|c|c|c|c|c|c|c|c|}
\hline 46 & 1 & Plastic & Fentanyl, Heroin, 4-ANPP & & & & & \\
\hline 47 & 1 & Plastic & Cocaine & 1.08 & & & & \\
\hline 48 & 1 & Plastic & Fentanyl, 4-ANPP & 0.25 & 1.08 & & & \\
\hline 49 & 1 & Plastic & Cocaine & 1.32 & & & & \\
\hline 50 & 1 & $\begin{array}{c}\text { Suboxone } \\
\text { Packet }\end{array}$ & Buprenorphine & & & & & \\
\hline 51 & 1 & Currency & Oxycodone & & & & & 19.00 \\
\hline 52 & 1 & Plastic & Cocaine & 6.39 & & & & \\
\hline 53 & 1 & Plastic & Cocaine & & & & & \\
\hline \multirow{2}{*}{54} & 1 & Plastic & Cocaine, Fentanyl, 4-ANPP & & & & & \\
\hline & 2 & Plastic & Cocaine, Fentanyl, 4-ANPP & 0.03 & 0.15 & & & \\
\hline 55 & 1 & Plastic & Buprenorphine & & & & & \\
\hline 56 & 1 & Plastic & Cocaine, Fentanyl & 0.12 & & & & \\
\hline \multirow{3}{*}{57} & 1 & Plastic & Methamphetamine & 0.09 & & & & \\
\hline & 2 & Plastic & Methamphetamine & 0.09 & & & 0.30 & \\
\hline & 3 & Plastic & MDMA & 0.40 & & & 1.50 & \\
\hline \multirow{3}{*}{58} & 1 & Plastic & Cocaine & 0.24 & & 1.14 & & \\
\hline & 2 & Plastic & Cocaine & 1.74 & 0.03 & & & \\
\hline & 3 & Plastic & Cocaine & 0.80 & & 0.30 & & \\
\hline \multirow{2}{*}{59} & 1 & Plastic & $\begin{array}{l}\text { Fentanyl, Heroin, 4-ANPP, } \\
\text { Etizolam, Deschloroetizolam }\end{array}$ & 0.18 & 0.15 & 1.62 & & \\
\hline & 2 & Plastic & $\begin{array}{c}\text { Heroin, Fentanyl, 4-ANPP, } \\
\text { Etizolam }\end{array}$ & 0.09 & 0.45 & 5.64 & & \\
\hline
\end{tabular}

